\title{
Hollywood on tobacco: how the entertainment industry understands tobacco portrayal
}

\author{
David L L Shields, Julia Carol, Edith D Balbach, Sarah McGee
}

\begin{abstract}
Objective-To determine how people in the California-based entertainment industry think about the portrayal of tobacco use in movies and on television. Specifically, to explore who decides when to include tobacco in a project; how that decision is made; what issues are considered; what messages are intended; whether and how the issue of secondhand smoke is considered; and what advocacy methods might be useful in influencing future decisions about tobacco portrayal. Design-Qualitative in-depth interviews of entertainment industry personnel,with a semi-structured interview protocol to guide the interview.

Subjects-54 subjects drawn from a convenience sample of writers, actors, directors, producers, studio executives, and others involved in the film industry.

Results-Hollywood is heterogeneous with varying perspectives on rates of tobacco use portrayal; intentionality of the decision to use and the necessity to portray tobacco use; and its degree of acceptance of responsibility for influencing societal smoking. Tobacco depiction may originate with the writer, actor, or director and is included most frequently to elucidate character or portray reality. On-camera smoking is influenced by actors' off-camera tobacco use.

Conclusions-The research presented can help advocates better understand the norms and values of those working within the entertainment industry and thereby assist them in creating more effective change strategies.

(Tobacco Control 1999;8:378-386)
\end{abstract}

Keywords: films; movies; television; tobacco use

American

Nonsmokers' Rights

Foundation, Berkeley, California, USA

D L L Shields

J Carol

S McGee

Tufts University, Medford,

Massachusetts, USA

E D Balbach

Correspondence to: Julia Carol, ANR

Foundation, 2530 San Pablo Avenue, Suite J, Berkeley, California 94702, USA;

juliac@no-smoke.org

Received 1 May 1999 Accepted 24 June 1999

\section{Introduction}

Adult smoking rates in the United States have declined substantially in recent decades. Awareness of the dangers of secondhand smoke has increased, and the number of public areas in which smoking is allowed continues to decrease, along with the social acceptability of smoking. ${ }^{1-3}$ In contrast to these changes, research has documented that smoking was three times more prevalent in movies than in the general population from 1960 to $1990,{ }^{4}$ and smoking in films and on television increased in the 1990s. ${ }^{5}$

This study was designed to learn how and why tobacco use is portrayed as it is on television and in the movies. Through interviews with writers, actors, directors, producers, and others involved in the television and movie industries, we investigated the Hollywood "culture" as it relates to on-camera tobacco use. Specifically, we were interested in who decides when to include tobacco in a project, how that decision is made, what issues are considered in making that decision, what messages are intended, whether and how the issue of secondhand smoke is considered, and what advocacy methods might be useful in influencing future decisions about tobacco portrayal.

\section{Method}

DATA COLLECTION AND ANALYSIS

During autumn 1997, we developed an open-ended interview protocol, tested it, and conducted 14 telephone pilot interviews with members of the film industry. Each interview was audiotaped, although only nine tapes were transcribed due to taping problems. The transcriptions were read by all members of the research team to identify a preliminary list of themes. In the main phase of the research (spring 1998), we conducted 37 additional interviews using a modified interview protocol. Interviews were conducted either by members of the research team or by an outside consultant who had greater access to film and television personnel. Each interview of 20 minutes to one hour duration was transcribed. From the transcriptions, we identified themes and used NUD*IST, a computer program designed to assist qualitative data analysis, to keep track of the themes through use of a coding scheme; this scheme was continuously revised and expanded in response to new interviews. We continued to conduct and code interviews until we reached theoretical saturation - the point at which little new information was being derived from additional interviews. All interviews were then re-coded by at least two members of the research team using the final coding scheme.

We then conducted a detailed textual analysis to find recurring themes, common assumptions, and points of interpretive disagreement and wrote summary statements for each code, attempting to encapsulate the various perspectives held by our interviewees. An independent facilitator presented a summary of our key findings to a focus group of eight members of the entertainment industry, none of whom had been interviewed previously. The focus group had the opportunity to confirm, disconfirm, or amplify the findings. This discussion was also transcribed, coded, and entered into the database. Finally, where significant differences of opinion were evident in our data, we 
Table 1 Professional experience of subjects by age and sex*

\begin{tabular}{lccccc}
\hline Age/sex & Actor & Writer & Director & Producer & Other \\
\hline <40 years & 7 & 3 & 3 & 4 & 4 \\
$\quad$ Male & 7 & 3 & 0 & 3 & 2 \\
Female & 5 & & & & \\
? years & & 8 & 3 & 5 & 4 \\
Male & 1 & 4 & 2 & 11 & 7 \\
Female & 5 & &
\end{tabular}

* Subjects may be included in more than one occupational category if they have had significant experience in more than one; however, we limited each person to no more than two primary occupational roles.

conducted computer runs to explore hypotheses about how these differences might relate to demographic characteristics.

We also reviewed television interviews with entertainment industry personnel, attended and reviewed the transcript of a political hearing that dealt with tobacco use in film, reviewed the relevant literature, and kept abreast of the work of others. This review of other source material helped us to confirm our findings.

INTERVIEWEES

All study participants were drawn from the California-based entertainment industry. To obtain the initial study participants, the American Nonsmokers' Rights Foundation mailed "contact forms" to its members and colleagues. In the second phase of the study, participants were obtained in multiple ways. Some were identified through the contact list built up during the first phase, some by third parties familiar with the research, and some by other study participants. Most, however, were contacted by a public relations firm hired to assist with the project. Every effort was made to obtain a broad cross-section of Hollywood.

Altogether, we analysed transcribed interviews with 54 members of the entertainment industry: 28 men and 26 women, ranging in age from 24 to 65 with a mean age of 40 . At the time of their interviews, nine were smokers, 13 were former smokers, 30 were nonsmokers, and two of unknown smoking status. Twenty interviewees worked primarily in the movie industry, 19 in television, and another 15 worked in both media (table 1).

\section{Results}

Our intent was to provide a description of the influence of Hollywood's "culture" on tobacco portrayal. In the course of our study, however, we learned that the industry is quite diverse, fluid, segmented, and heterogeneous, with people frequently shifting occupational roles. There is a substantial difference between the norms and practices of the film and television industries. The television industry appears to be more hierarchical, structurally stable, and internally integrated than does the film industry. Perhaps because television programming is carried across the public airways and must seek corporate advertising, it tends to be more "mainstream" in its content and values.

As a result, in reporting these results, we identify people by occupation and by media and try to capture the diversity of the industry. We present our findings under 10 main headings and conclude with comments about the implications of our study for tobacco control.

RATES OF PORTRAYAL

Research suggests that smoking in the movies has increased over the past decade. ${ }^{5}$ People's impressions, however, may differ from objective rate counts. When asked about trends in on-camera smoking, several interviewees spontaneously compared current rates to the "classic" periods of film and television, suggesting that there is less smoking now on television than in the '50s and '60s and less in film than in the '30s and '40s. They uniformly attributed the decline to greater public awareness of the health hazards of smoking and a social trend away from approval of tobacco use.

"Look at films in the '30s and '40s. Everyone had a drink in one hand and a cigarette in the other. That was part of the culture and it was supposed to be glamorous. And it was also because actors needed props to hold in their hand and that's still the case. But I think there's probably in the last 10 years been a lot less smoking and I think that has a lot to do with the actors. As much as anything else, they just try not to smoke like everybody else who's stopped smoking."

Female, producer, film

When asked specifically about the trends in the past decade or so, the answers were quite different for television and film. Most thought there had been a decrease on television, though several had not noticed any change; only one thought smoking had increased. Those who thought there had been a decrease attributed it to the networks' standards and practices (a set of guidelines about what may be depicted on-camera), the quick pace at which television scenes move, the general unpopularity of smoking among the public, the need for main characters to be likeable, and to the California law requiring most workplaces, including television production sets, to be smokefree.

Those interviewed were much more divided over recent trends in film. Most thought there had been a recent increase, which nearly everyone attributed to an increase in independent films and the popularity of gritty images. Several also noticed an increase in smoking in films targeting audiences under 40 .

"It seems like there's an increase in smoking in films, especially with 20-somethings, 30somethings ... In a lot of the independent [films] ... it's almost like if they're not smoking, it's unusual.”

Female, producer, television Several interviewees thought there had been a decrease recently, and several had not noticed a change either way. When told of the research indicating an increase, those who had felt there had been a decrease were inclined to question the science of these reports.

\section{PRODUCT PLACEMENT}

Before 1989, it was common for tobacco companies to pay to have their products included in entertainment programs. ${ }^{6}$ Sylvester Stallone, for example, agreed to use Brown and Williamson tobacco products in no less than 
five feature films for a fee of US $\$ 500000 .^{7}$ In response to widespread criticism, in 1989 the industry voluntarily agreed to stop all paid tobacco product placements, although internal tobacco industry documents suggest that placements may have continued well into the 1990s. ${ }^{8}$ For cigars, paid product placement continued at least until $1998 .^{9}$

No interviewee spontaneously suggested paid product placement as a reason for why tobacco use is included in entertainment media products. When queried about it directly, however, many of our interviewees said they thought it occurred, although none had any first-hand knowledge of it. A typical comment:

"I'm sure it exists. I don't want to believe it exists, but I know how much product placement there is in the world, and I believe that the tobacco lobby is powerful and strong and rich."

Male, producer, film

No one thought product placement played a prominent role in determining whether tobacco use would be depicted. Rather, respondents believed that if smoking was to be depicted, then either placement deals or product donations might determine the brand, particularly among independent film producers:

"They [independent producers] charge most of their post production on their credit cards. So they're happy to get a bone from wherever they can get a bone. And if that means putting a pack of Marlboro on a table next to a major character for a couple of seconds on screen, you're gonna do it."

Female, actor, television

Virtually everyone agreed that there was no product placement in television. In fact, several commented on the extent to which networks go to eliminate mention of brand names.

WHO MAKES THE DECISION?

When asked who is responsible for deciding to use tobacco, there was broad agreement that it could be any of several people.

"It could start with a scene direction in the script that could be 'character smoke'. And it can be the actor, once they're on the set, deciding that they want their character to smoke or not smoke ... It could be the director deciding that."

Female, producer, film

Writers, actors, and directors are most likely to initiate tobacco depiction, with actors the single most commonly identified source. The final decision, however, reflects input from all of these sources, with perhaps additional input from the camera operators. When differences of opinion arise, it is often the person with the most clout who prevails:

"If Arnold Schwarzenegger . . . says, 'I'm gonna go smoke a cigar,' I don't think there's anyone in the world who's gonna tell him, 'No, you're not."' Male, producer, film/television

Although these comments apply to the movies and television, we found important differences between them. Within the movie industry, producers and studios are not seen as playing a major role in the creative development of a project. In television, however, studios play a much more prominent role, often through formal guidelines. Moreover, in television many of the producers are also writers, which increases their influence in the decision-making process. As one respondent observed, "In movies the director is king or the star is king, but in television it's very much a producer's medium because the producers are usually the writers."

\section{WHY IS TOBACCO USED IN FILM?}

The reasons for tobacco depiction generally fell within two categories. Some reasons such as character development stem from the internal logic of the project itself. These reasons are connected to artistic judgments made by those responsible for the creative development of the project. Other reasons (such as if an actor personally smokes) are more external to the artistic process.

\section{Intrinsic reasons for portrayal of tobacco use}

By far the most frequently cited reason for tobacco depiction was that it helped to develop or reveal some aspect of the character:

"If a character is slightly manic or out of control or nervous, you can visualize that very clearly by people having cigarettes in their hands and ... you can create visually a tremendous sense of tension or conflict or whatever by having people use a cigarette and, also, by the way they smoke it, whether it is with a certain kind of desperation or intensity."

Male, director, film

Most industry personnel see the cigarette as a flexible prop that can be invested with a variety of meanings and messages. It can help create an aura of sexiness and sophistication, for example, or convey an image of someone caught in the grip of addiction, self-abuse, or neurosis. In fact, when asked what characteristics an actor might convey through smoking, several interviewees had trouble answering, not because it was difficult to think of any, but because the possibilities were so expansive, ranging from positive to negative. Rebelliousness, recklessness, "coolness", toughness, weakness, nervousness, indifference, and social inferiority were most frequently mentioned. The full range of possibilities, however, was more evident among those in the film industry. Most who worked in television felt smoking was almost always associated with negative character traits, because networks would not allow positive images of smoking.

There are also meanings or images that can be conveyed now through a cigarette that were not available in previous film eras. As two interviewees noted, the contradiction between what we now know about the health effects of tobacco and the reality of continued use open up new possibilities for character depiction. Smoking can now communicate such personal qualities as inner dissonance or psychological inconsistency.

"There was that character in [name of movie] who would go for a jog and at the end of the jog he'd light up a cigarette. That's a good character illustration because he was a man of paradoxes. He would do something good on one hand and then something bad on the other. And he did that in his professional life, his romantic life, and in his health life as well."

$$
\text { Male, director, film/television }
$$


Though few interviewees saw variation in the meaning of a cigarette by the race/ethnicity of the smoker, many commented on variation by age and sex. In particular, a smoking teenager might convey an image of rebelliousness, whereas an older smoker is more likely to convey an image of addiction, self-destructiveness, or lack of control. Our interviewees felt female smokers were more often sexy and sophisticated, while images of toughness, "coolness", and nervousness applied equally to both sexes and all ages. Cigars were often used to depict status or trendiness.

In addition to character development, our respondents suggested other reasons for tobacco depiction. Tobacco use might be included in a film or scene to enhance its realism. Specific groups, such as high school dropouts, historical periods, such as mid-century, or locations, such as a bar, would not seem real-many of our interviewees suggestedwithout the presence of smoking. A cigarette may also provide the actor with something to do with his or her hands, or enable physical movement from one part of the set to another. Finally, smoking is sometimes included for the pure lighting effect of the on-camera smoke.

\section{Extrinsic reasons for portrayal of tobacco use}

Not all reasons for inclusion of smoking have to do with artistic judgments. Two interviewees mentioned that tobacco is included because it is within the historical tradition of cinema. Filmmakers also may see cigarettes as a way to project an edgy or gritty image, thus enhancing box office appeal. Several interviewees commented on how independent films in particular seek to be "edgy", targeting a market that either is or would like to be "counter-culture". In contrast, television studios have more of a corporate culture and desire to project a trendy, yet mainstream, image.

The most significant cause of smoking on-camera apart from the artistic reasons, however, is simply that many actors smoke in real life. While half of the directors, writers, and producers did not believe that the personal tobacco-use habits were important, actors themselves were virtually unanimous in acknowledging the relationship. One actor commented:

"I'm a smoker and my character study is every character I play smokes a different brand of cigarettes."

Female, actor, film

HOW DELIBERATE IS THE DECISION?

With smoking being the single greatest cause of death in the United States, and exposure to secondhand smoke the third leading cause, ${ }^{10}$ one might anticipate that decisions about its portrayal on film would be given careful thought. The care taken in making these decisions, however, seems to vary widely. According to some of our interviewees, generally little thought is given to tobacco depiction. As one producer commented, "I don't think it's really that careful of a decision; I think it just happens the way anything happens when you're telling a story."
Others, however, felt differently. They suggested that it was nearly impossible not to give careful thought to tobacco depiction, because it is so much in the public consciousness and because it causes problems in filming and editing. According to one actor:

"There's nothing arbitrary about smoking on film. ... It's a deliberate choice. It's very hard to smoke in a movie ... because every take you have to light up and be at the same spot you were. It's a big commitment to smoke on film. So in a scene that on film may take two minutes can take three hours, and you're lighting up every time you do a take, and it's hard."

Female, actor, film

In addition to technical difficulties, smoking on film has come to be seen as a problem by many in film and television. For them, the decision of whether to include smoking depiction is no longer made on purely artistic grounds. It has become, in part, a moral issue. As one person commented:

"[Previously,] whether the character smoked or not was not discussed from the standpoint of what would be responsible as filmmakers but what was appropriate to the character. Now we think about it more in the other way, in both ways."

Female, producer, film

TOBACCO DEPICTION: HOW ESSENTIAL IS IT? We asked our interviewees whether they considered tobacco depiction to be a problem and whether they considered it essential. In order of frequency, respondents fell roughly into three categories: (a) those who found it useful, but rarely necessary, and thus somewhat of a problem; (b) those who considered it to be gratuitous and highly problematic; and, (c) those who believed on-camera smoking to be quite useful and not a problem.

Most of our interviewees fell into the first category-those who felt tobacco depiction might have negative social repercussions, but who still felt its usefulness as a communication device took precedence in some instances. Many of these people volunteered that cigarettes are overused props that sometimes substitute for good acting. For example, one person, commenting on Julia Roberts in $M y$ Best Friend's Wedding, said:

"I felt that [her] smoking was extremely distracting to me as an audience member; I thought it was not great acting. . . I thought it was a prop that was unnecessary and I think it took me out of her character a lot because I felt she was leaning on it as a prop and not making a statement about the character."

Female, producer, film Smoking was not just perceived to function as a crutch for actors, but for writers as well:

"I know that in my earlier screenplays, having a character smoke was often a crutch. .. .It's a shorthand to character. And I think that over the last few years, people have gotten away from that crutch because: (a) when you read any book on screenwriting or go to any seminar on screenwriting, one of the first things they say is, "Stop using the cigarette crutch; stop using the telephone crutch; stop using the driving around in a car crutch." And I think it's become cliché to the point where people don't use it as much." Male, producer, film/television 
Many of these interviewees resolved the tension by limiting tobacco depiction to characters with negative traits or characters who eventually get sick from it. The assumption seemed to be that viewers would be less inclined to imitate the behaviour of those portrayed in a negative light.

"I think when a character is a villain and an evil character and they're unattractive in ways, the smoking isn't as glamorous."

Male, producer, film

Those in the second category, who were strongly committed to non-depiction of tobacco use, believe it to be nearly always inappropriate and gratuitous.

"As far as I'm concerned, cigarettes are literally a smokescreen between you and the audience and the character that they're portraying."

Male, cameraman, film

Finally, there were a few who saw no difficulty whatsoever with tobacco depiction. Coming mostly from the film industry, they felt that their job was to depict reality.

"I don't personally think that positive imagery in the media is necessarily a good thing. I think honest depiction is more important. I think false positive images are just as harmful as negative images because they're bullshit and they're bad for the psyche."

\section{Male, writer, television}

REASONS FOR NON-DEPICTION

We asked our interviewees about nondepiction as well as depiction. Some of the answers simply reflected the flip-side of the reasons for portrayal. For example, the theme "It just didn't fit the character" was echoed in numerous interviews. Many commented on cigarettes being overused props. Some observed that a few film studios, most notably Disney, have a cultural identity such that people working for them know, even without explicit guidelines, that most tobacco depiction is inappropriate.

Many television studios have specific policies or guidelines that restrict the portrayal of tobacco use. For example, CBS program standards state:

"Character portrayals and scenes depicting the consumption of alcohol, drugs, cigarettes and similar substances must be thoughtfully considered, essential to plot and role development and not glamorized. When the line is crossed between normal, responsible consumption of a particular substance and abuse, the distinction must be clear and the adverse consequences of abuse specifically noted and explored."

Some of our interviewees, mainly from television, had been involved in projects where either they or someone else on the project intentionally included anti-smoking content. Anti-smoking messages were conveyed either by associating smoking with negative personality traits or by driving home the adverse health consequences of tobacco use.

"You show somebody who's ravaged over years by nicotine. And then has emphysema, the last stages of emphysema, and those are pretty frightening images."

Male, writer, television
The California law that bans smoking in indoor work areas has also had an inhibiting effect, according to several of our interviewees, on the portrayal of smoking on television.

Within the movie industry, non-depiction usually occurs for one of two reasons. Non-depiction may occur naturally because smoking just does not fit the theme, characters, or circumstances. Alternately, tobacco use may be absent because of the strong personal beliefs of an influential writer, actor, director, or producer.

Two interviewees made the point that smoking is not missed, even when the project's theme could have easily lent itself to the inclusion of smoking.

"I don't think that anybody who saw that movie stopped and said, "Gee, nobody's smoking in that movie! I wonder why they're not smoking?" And, you know, that's what I think - people won't miss it if they don't see it."

Female, producer, film

INDUSTRY RESPONSIBILITY

We asked respondents about the extent to which Hollywood shaped society and the extent to which it was shaped by society. The question was framed generally, without specific mention of tobacco. As expected, most interviewees began by acknowledging a reciprocal influence between society and the entertainment industry.

"All you have to do is look at the hairstyles that you see on young women to see evidence that Hollywood influences behavior. . . B But then on the other hand, certainly what the public responds to and watches determines what programming gets made."

Female, studio executive, television

The easy consensus around a reciprocal influence model of the relationship between the entertainment industry and broader culture quickly dissolved, however, when questions turned to issues of responsibility. At that point, many of our interviewees took a more unidirectional view of influence, with some emphasising the media as mirror motif and others, the media as shaper motif. Thus, when asked whether watching characters smoke influenced viewers to smoke, some respondents minimised their responsibility:

"I believe that films always mirror society; what film makers are trying to do [is] to document what's happening in society. . . . I think that if people are going to be influenced to smoke less or to start smoking or smoke cigarettes as a consequence of watching motion pictures or television, then they probably need more help than anyone can give them."

Male, director/producer, television

Similar comments were echoed by several respondents who indicated that tobaccobecause it is a less serious issue in their minds than other issues, especially violence-does not rise to a level deserving of special attention.

Although a minority, other interviewees saw themselves as highly responsible for trends in society, including the trend towards smoking among teenagers. They took this responsibility seriously and often wondered what they could do to improve the situation. 
"We have to take matters into our own hands, as the architects and custodians of pop culture, to really police ourselves."

Male, producer, film

A few of our interviewees reflected on the ambiguity inherent in visual images. Although they sought to be responsible in their work, they also recognised their limitations, understanding that they had little control over how it is appropriated.

"As a filmmaker you never know what a viewer will appropriate from the image you create, regardless of what your intention was in creating that image. You have to be very careful because ... a picture tells a thousand words and they aren't all yours."

$$
\text { Female, producer, film }
$$

HOW BEST TO APPROACH THE INDUSTRY When we originally conceptualised this research project, the possible relationship between smoking on-screen and rising rates of teenage smoking was only rarely, if ever, a topic of conversation within either the entertainment or tobacco control communities. Our original intent in this project was to "listen" before "acting", because sound advocacy is built on a clear understanding of the community targeted for change.

However, smoking in movies became a highprofile topic with the proposed "global settlement" of tobacco litigation in spring 1997. Smoking in the movies was discussed on the Senate floor, and Hillary Rodham Clinton ${ }^{11}$ and United States Vice President Al Gore ${ }^{12}$ criticised the industry. California State Senator John Burton held hearings on the issue with key industry leaders. ${ }^{13}$ In addition, the Entertainment Industries Council launched a high profile initiative to reduce the gratuitous use of tobacco in film. ${ }^{14}$

We considered it likely that some of our interviewees might have been influenced by the public attention given to the issue. Moreover, as they were the targets of high profile efforts to modify behaviour, we felt that the controversy might have affected their thoughts on what advocacy methods might be effective. To gauge these possibilities, we asked interviewees about their awareness of the controversy surrounding smoking on-screen, and the extent to which the topic had become a point of discussion among their colleagues. Surprisingly, nearly half of our respondents were unaware that tobacco use depiction had become a controversial issue. Among those who were aware of the public scrutiny that had been focused on smoking in the media, responses were varied. Although some did not feel that it had had much of an impact, some, particularly from the movie industry, were angered by the attempt of politicians to influence their work, with many believing that tobacco use was a minor issue:

"I think that's the return of fascism basically. ... I think it's a violation of our rights and it's, to me, very frightening as a society to allow that to happen."

Male, producer, film

At the other end of the continuum, some, mostly from the television industry, felt that the outside pressure to change the portrayal of smoking was beneficial. The following comment is illustrative:

"I think it would be extremely naive to think that much is going to really change in the portrayal of smoking if there isn't some pressure greater than public opinion, some vocal pressure to cause people to say, 'Whoa, we've got to get our act together on this or else we're gonna be looking at regulation'. The whole history of our industry suggests that it's the threat of regulation that causes us to act. I'm not proud of it, but that's it." Female, studio executive, television

When we asked our interviewees about how best to approach the entertainment industry, the answers were again quite varied. With a few notable exceptions, most in the motion picture industry felt that neither outside pressure nor internal top-down pressure would work, and might even be counterproductive. Most interviewees laced their responses with caveats about artistic freedom. In fact, several interviewees felt that simply posing the question of influencing the industry raised the spectre of censorship.

"I would never presume to censor or to recommend censorship or to create some kind of watchdog... I mean look at Standards and Practices. It's a disaster; it's not good."

Female, producer, film

Several of our respondents suggested that the "creative personality" is highly reactive to pressure, often resulting in the opposite of what is desired.

"I just think that everyone has to be careful not to be dictatorial about these issues because that just makes peoples' hackles rise. You know the minute you tell some of these filmmakers, who all think they're infants terribles anyway, that they can't do something, they'll turn around and spit in your face and say, 'I'll do what I please,' even if they agree with you."

Female, studio executive, film

Although some within the television industry were also resistant to any outside or top-down pressure, they were more open to hierarchical structure and guidance from studios. In fact, many looked for leadership from the upper echelons of their profession.

"I think the best way is probably to reach the individual producers of each show that's actually on the air. . . . If you get to the actual writers/producers of the shows, it's probably the most effective way, because they're the ones that are creating the material."

Female, actor, television

Finally, several interviewees felt that the best way to reach the industry may not be to approach the industry directly, but to continue to make progress on the issue within the general population. They suggested that the public education campaigns about smoking had already influenced the industry and would continue to do so. A number of our interviewees mentioned various advertising campaigns put out by the California Department of Health Services, as well as state laws banning smoking in certain venues, as having had an impact on how the entertainment industry deals with smoking. 


\section{Discussion}

In summary, Hollywood is heterogeneous with varying perspectives on the rates of tobacco use in movies and on television, the necessity of portraying tobacco use, and Hollywood's degree of responsibility for societal smoking. Perceptions also varied on how conscious the decision was either to use or not use tobacco. The decision to portray tobacco use may originate with the writer, actor, or director, and tobacco is included most frequently to elucidate character or portray reality. On-camera smoking is influenced by actors' off-camera tobacco use.

How then should these results inform the efforts of tobacco control advocates? Health advocates are currently actively encouraging a reduction in the depiction of tobacco use in film, believing that such a reduction will help to prevent or reduce smoking among the general public, especially minors. Some advocates will use an "inside" strategy, working within the entertainment industry. Others will use an "outside" strategy, one which focuses the public spotlight on the issue and creates social pressure for change. The present investigation uncovered information that can help guide advocacy efforts, whether advocates are using an "inside" or an "outside" strategy. We have distilled seven key issues to consider when devising advocacy strategies.

WE NEED A MULTI-PRONGED APPROACH

One of the most important things that we learned is that the industry is quite diverse, fluid, segmented, and heterogeneous, making it close to meaningless to talk about "the entertainment industry" or "the Hollywood culture". One implication this finding holds for advocates is that they will have a difficult time finding ways to reach more than small segments of the industry. Even those who share a similar occupational role-producers, for example - do not interact or communicate frequently with others in their guild. A sustained, coordinated, multi-channel approach is clearly warranted and needed.

INCORPORATE EDUCATIONAL STRATEGIES

Education of those within the industry can play an important role, because it was clear from the interviews that many within the industry had not seriously thought about tobacco. Many of our interviewees, and particularly members of the focus group, spontaneously commented on how enlightening it had been to discuss the issue in the course of the interview.

Educational modes of advocacy are potentially useful for "inside" and "outside" strategies. On the "inside", education is a way to gain the voluntary cooperation of entertainment industry personnel. For example, the Directors, Writers, and Actors Guilds might be conduits to people working in the industry and help to encourage them to think about the impact of including tobacco use before they do so. Working "outside", advocacy groups can educate their own constituents about the nature of the entertainment industry and can continue to work on norm changes among the public. For example, nearly all of our interviewees believe that as the broader culture has changed in its views about smoking, the industry has generally followed suit.

COMMUNICATE AN ANTI-CENSORSHIP POSITION Many within the industry preferred educational strategies because such strategies avoided the "briar-patch" of censorship. Those working within the film industry were particularly protective of artistic freedom, and effective influence strategies can be designed to affirm that value. The need to support artistic freedom places a burden on tobacco advocates to communicate clearly that we do not want censorship, but want instead tobacco depiction only when a project would substantially suffer without it.

Although film and television personnel abhor censorship, those in television already work under guidelines. Moreover, some interviewees thought that their colleagues would not change their behaviour unless they perceived a credible threat of regulation. Those working the "outside" strategy may need to finds ways to create such a threat, so that those working an "inside" strategy can create internal momentum for voluntary change.

IF YOU HIT HARD, HIT SMART

Tobacco control needs members of the entertainment industry as allies. Advocates intent on an "outside" hard-hitting campaign need to craft their message to provide an uncomfortable, but still potentially constructive, stimulus for change. It may backfire, for example, to blame the entertainment industry for the rise in teenage smoking. Such an attack can easily be deflected as simplistic, as broad social trends such as teenage smoking rates stem from complex webs of causal factors rather than single sources. Pointing to instances when the cigarette is used in a superficial, unnecessary, or clichéd way, and challenging writers, actors, and directors to find other, better vehicles of communication, can provide a professional challenge that still affirms their competence and creativity.

ADDRESS MULTIPLE OCCUPATIONAL GROUPS

Smoking portrayal may originate with writers, actors, or directors, although actors seem to be the most frequent source. This finding points to the necessity of working with multiple segments of the entertainment industry. As actors are the single most likely source, they may seem to be the most appropriate audience for tobacco control advocates to address. On the other hand, apart from top box-office stars, actors often feel like they are at the bottom of the power pyramid. Although directors and producers are less likely to initially suggest tobacco use, they are more likely to have the power to determine the final outcome. Some producers, for example, simply do not allow smoking in their films.

WORK TO REFRAME THE ISSUE

We found that on-camera tobacco portrayal occurs for three main reasons: (a) cigarettes are 
a means through which an actor can convey information about a character; (b) people smoke in real life and film-makers are concerned about reflecting that reality; and (c) actors who smoke in their everyday life often prefer to act with a cigarette in hand. Each of these reasons provides openings for tobacco control advocates.

Many writers and actors recognise that conveying character information through smoking is often a "lazy" reason for inclusion of tobacco use, a fact that is used in media-advocacy efforts by the Florida "Truth" campaign. ${ }^{15}$ For example, one newspaper advertisement created an "L" rating for movies where the movie-makers were too "lazy" to find a better way than cigarettes to make a character appear rebellious or sexy. This may offer a model of advocacy with the entertainment community.

Accepting realism as a value, the tobacco control community has already begun to engage the entertainment industry in a dialogue about improving on their realism. ${ }^{16}$ The discussion ought to continue and expand. How realistic is the smoking portrayed? Do portrayals of police officers who smoke, for example, include the image of them unable to run down a suspect because they are short of breath? For example, smokers in the movies were more intelligent and more romantically and sexually active than non-smokers, often ignoring the actual negative correlates of smoking. In addition, the negative consequences of smoking were rarely presented in the movies. ${ }^{17}$ Realism around the issue of secondhand smoke is also a relatively unexplored area for the entertainment industry. In real life, when people light up in the middle of a crowd, a number of people around them are annoyed and may wave the smoke away. In fact, interesting visual tension might be productively conveyed using response to secondhand smoke as the stimulus.

Actors might be influenced to reduce on-screen smoking through education. Smokers, particularly younger smokers, overestimate the percentage of people who smoke in reality. ${ }^{18}$ So it is plausible that actors who smoke are likely to overestimate the likelihood that a particular character would smoke. In addition, half of the writers, directors, and producers were unaware of the tendency of smoking actors to portray smoking characters. Simply raising their awareness on this point may lead them, at least in some instances, to question the decision of the actor to portray his or her character as a smoker.

Keeping all three of these motives for inclusion of tobacco portrayal in mind, health advocates ought to direct the discussion of smoking depiction to the central question of necessity. Simply pointing to the plausibility of a cab driver being a smoker, for example, is not a high enough bar to set for acceptable smoking on screen. The question is whether a particular scene would lose its desired communicative effect if the tobacco depiction were removed. Framing the question in this way may help the industry adopt a norm to include tobacco depiction only when the scene would suffer substantively without it.

HELP THE INDUSTRY CREATE EFFECTIVE

ANTI-TOBACCO MESSAGES

To date, when members of the industry have included anti-tobacco content, they have largely relied on one or the other of two methods. The first involves portrayal of negative health consequences stemming from tobacco use, an approach reminiscent of the 1960s health education models that were discarded because of beliefs about their ineffectiveness. ${ }^{19}$ The industry also has tried to convey an anti-smoking message by having smokers portrayed in a negative light. However, even when smoking is designed to convey that a character is flawed, the character may still have considerable appeal to certain viewers. Recent research on effective tobacco control message ${ }^{20}$ might be of interest to many within the entertainment industry. Creative treatments of tobacco industry manipulation and secondhand smoke themes, for example, might well play an important role in reducing the allure of tobacco.

\section{Conclusion}

In conclusion, there is no "quick fix". While the pro-health community has gained some momentum in its commitment to working to reduce tobacco depiction in movies and on television, it needs a well-thought out approach. Moreover, given the nature of the entertainment industry, it is critical that all advocacy strategies be not only well-planned, but coordinated. In the effort to create change, an "inside" strategy and an "outside" strategy may have important roles to play. Although the "inside" strategy of working with the industry, rather than against it, has great potential for effecting change, it may need the spur of the outside strategy to keep it moving forward.

The HOT (Hollywood on Tobacco) Project is a project of the ANR Foundation and is made possible by funds received from the Tobacco Tax Health Protection Act of 1988-Proposition 99, under Grant Number 96-26631 with the California Department of Health Services, Tobacco Control Section.

1 US Centers for Disease Control and Prevention. Surveillance for tobacco-use behaviors-United States, 1990 1994. MMWR CDC Surveillance Summaries 1994;Nov 18.

2 Fishman JA, Allison M, Knowles SB, et al. State laws on tobacco control-United States, 1998. MMWR 1999; 48(SS03): 21-62.

3 US Centers for Disease Control. Passive smoking: beliefs, attitudes, and exposures-United States, 1986. MMWR 1988;37(15):239-41.

4 Hazan AR, Lipton HL, Glantz SA. Popular films do not reflect current tobacco use. Am f Public Health 1994;

5 Stockwell TF, Glantz SA. Tobacco use is increasing in popular films. Tobacco Control 1997;6:282-4.

6 Luken TA. Testimony at hearings before the Subcommittee on Transportation and Hazardous Materials, Committee on Energy and Commerce, US House of Representatives, 101 st Congress, 1st session, 25 July and 13 September 1989, Serial No 101-85. Washington, DC: US Government Printing Office, 1989;1-3.

7 Glantz SA, Slade J, Bero LA, et al. The cigarette papers. Berkeley: University of California, 1996;364-74.

8 Maresca GP. The American Tobacco Company letter to Cliff McMullen (President, Unique Product Placement, Inc.) RE: Contract Renewal, 1992; Bates - N ATX040161292.

9 Klein A. Cigars lose starring role in future movies, TV shows. Baltimore (Maryland) Sun 1998 Mar 21.

10 Glantz SA, Parmley WW. Passive smoking and heart disease. Epidemiology, physiology and bichemistry. Circulation 1991;83:1-12 
11 Rodham Clinton $\mathrm{H}$. Hollywood role models promote lung cancer. San Francisco Examiner 1997 Aug 6.

12 Anon. Hollywood taking heat for all the lighting up in films. USA Today 1997 Aug 25.

13 Vanzi M. Lawmaker tar 25 . ence youngsters to light up, says state Sen. John Burton, ence youngsters to light up, says state Sen. John Burton, who will hold a hearing in effort to heighten awareness

4 among filmmakers. Los Angeles Times 1997 Oct 26:A-3. Madigan N. Hollywood alliance denounces tobacco use.

Truth Campaign, Florida Tobacco Pilot Program, Tallahassee, Florida, 1998

16 Next Generation, California Tobacco Control Alliance, Entertainment Industry Working Group, Mission Statement, Sacramento, California, 1997.
17 McIntosh WD, Bazzini DG, Smith SM, et al. Who smokes in Hollywood? Characteristics of smokers in popular films from 1940 to 1989 . Addict Behav 1998;23:395-8.

18 US Department of Health and Human Services. Preventing tobacco use among young people. A report of the Surgeon General, 1994. Atlanta, Georgia: Public Health Service, Centers for Disease Control and Prevention, Office on Smoking and Health 1994. (US Government Printing Office Publication No S/N 017-001-00491-0.)

19 Lynch BS, Bonnie RJ. Growing up tobacco free: preventing nicotine addiction in children and youth. Washington, DC: Institute of Medicine (National Academy Press), 1994.

20 Goldman LK, Stanton A. Evaluation of anti-smoking advertising campaigns. $\mathcal{F} A M A$ 1998;279:772-7.

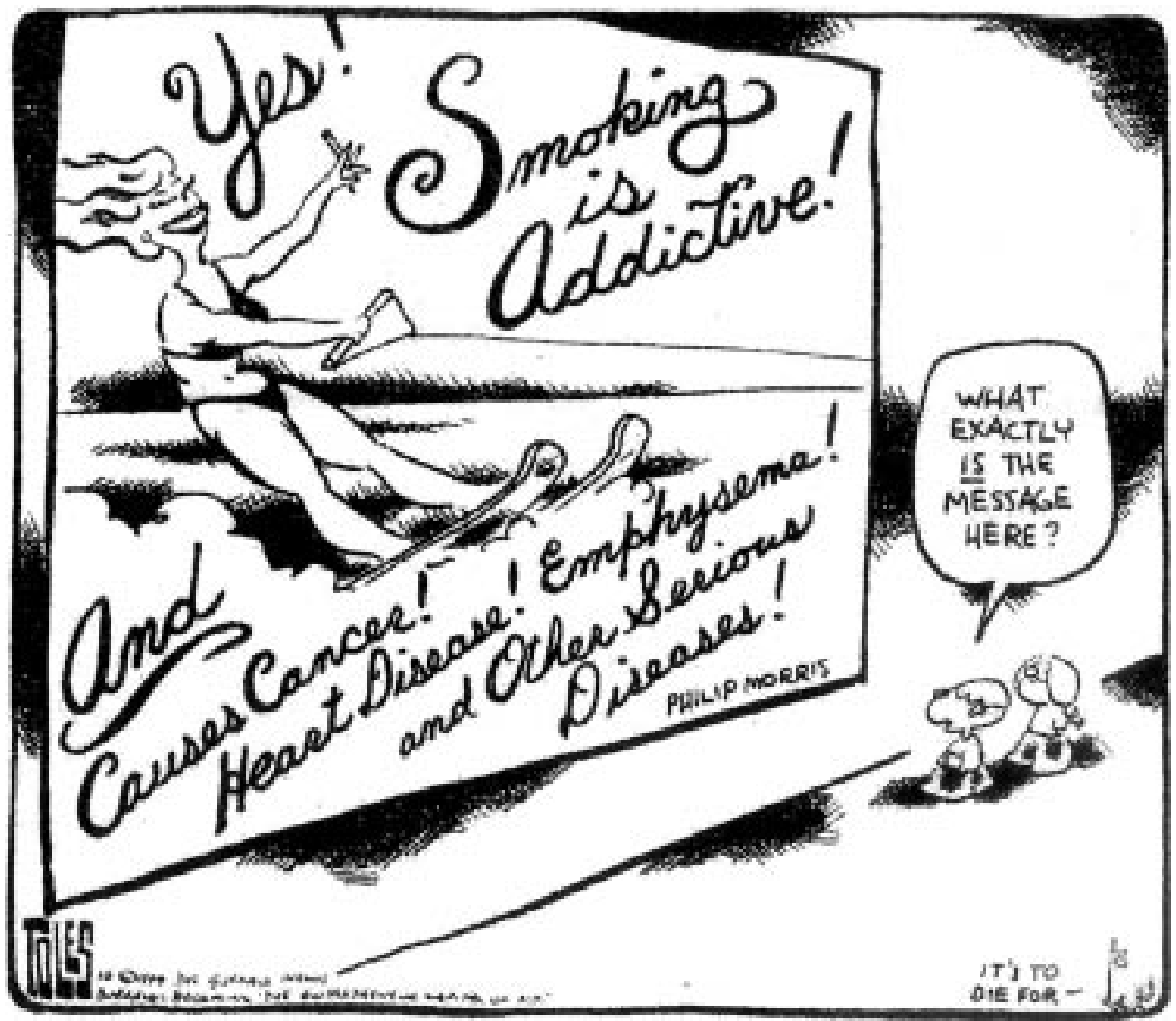

TOLES@1999 The Buffalo News. Reprinted with permission from Universal Press Syndicate. All rights reserved. 